\title{
Deletion of Tmtc4 activates the unfolded protein response and causes postnatal hearing loss
}

\author{
Jiang Li, ${ }^{1}$ Omar Akil, ${ }^{2}$ Stephanie L. Rouse, ${ }^{2}$ Conor W. McLaughlin, ${ }^{2}$ Ian R. Matthews, ${ }^{2}$ Lawrence R. Lustig, ${ }^{3}$ Dylan K. Chan, ${ }^{2}$ \\ and Elliott H. Sherr ${ }^{1,4}$ \\ 'Department of Neurology and ${ }^{2}$ Department of Otolaryngology - Head and Neck Surgery, University of California San Francisco (UCSF), San Francisco, California, USA. ${ }^{3}$ Department of Otolaryngology - \\ Head and Neck Surgery, College of Physicians and Surgeons, Columbia University and New York Presbyterian Hospital, New York, New York, USA. ${ }^{4}$ Department of Pediatrics, Institute of Human Cenetics, \\ Weill Institute for Neurosciences, UCSF, San Francisco, California, USA.
}

\begin{abstract}
Hearing loss is a significant public health concern, affecting over $\mathbf{2 5 0}$ million people worldwide. Both genetic and environmental etiologies are linked to hearing loss, but in many cases the underlying cellular pathophysiology is not well understood, highlighting the importance of further discovery. We found that inactivation of the gene Tmtc4 (transmembrane and tetratricopeptide repeat 4), which was broadly expressed in the mouse cochlea, caused acquired hearing loss in mice. Our data showed Tmtc4 enriched in the endoplasmic reticulum, and that it functioned by regulating $\mathrm{Ca}^{2+}$ dynamics and the unfolded protein response (UPR). Given this genetic linkage of the UPR to hearing loss, we demonstrated a direct link between the more common noise-induced hearing loss (NIHL) and the UPR. These experiments suggested a novel approach to treatment. We demonstrated that the small-molecule UPR and stress response modulator ISRIB (integrated stress response inhibitor), which activates elF2B, prevented NIHL in a mouse model. Moreover, in an inverse genetic complementation approach, we demonstrated that mice with homozygous inactivation of both Tmtc4 and Chop had less hearing loss than knockout of Tmtc4 alone. This study implicated a novel mechanism for hearing impairment, highlighting a potential treatment approach for a broad range of human hearing loss disorders.
\end{abstract}

\section{Introduction}

Sensorineural hearing loss, the most common cause of hearing impairment, can result from genetic mutations, noise exposure, aging, or other environmental exposures, including ototoxic drugs such as aminoglycosides and cisplatin. These ototoxic injuries typically share, as their end result, damage and reduction in synapses between sensory hair cells and afferent nerves, apoptotic death of hair cells themselves, and subsequent regression and degeneration of auditory neurons and cochlear supporting cells $(1,2)$. A number of organ- and cellular-level mechanisms have been implicated in noise-induced hearing loss (NIHL) (3). These include mitochondrial oxidative stress, in which reactive oxygen species are released in the cochlea upon noise exposure; $\mathrm{Ca}^{2+}$ overload in hair cells due to excessive entry through ion channels (4); and mitochondrial dysfunction and ischemia leading to ATP depletion (5).

There is currently no effective medical treatment for acquired or genetic sensorineural hearing loss. Small-molecule antioxidants such as $\mathrm{N}$-acetyl cysteine (6) and ebselen (7), a peptide inhibitor of the Jun kinase (8), and L-type $\mathrm{Ca}^{2+}$-channel blockers $(9,10)$, have been shown to prevent hearing loss in ani-

Conflict of interest: EHS and DKC are founders and shareholders in Jacaranda Biosciences. EHS is listed as an inventor on the published patent application, novel methods of treating hearing loss (patent cooperation treaty/US2016/058348)

License: Copyright 2018, American Society for Clinical Investigation.

Submitted: September 25, 2017; Accepted: August 30, 2018

Reference information: J Clin Invest. 2018;128(11):5150-5162.

https://doi.org/10.1172/JCI97498. mal models; however, efficacy in human trials has thus far been limited $(11,12)$. Many of these therapeutics have targets far downstream in the terminal pathways of oxidative stress and apoptosis, which may explain this lack of efficacy. Identification and modulation of additional pathways involved in genetic and acquired hearing loss, particularly those more upstream and proximal to the mechanism of initial cochlear trauma, would be invaluable to reveal new targets for pharmacologic and biologic therapy. Indeed, work by our group and others on genetic animal models of congenital and noise-induced deafness owing to deficiency in VGLUT3 (13) and pejvakin (14), respectively, has led to demonstration of the potential utility of gene therapy in hearing restoration in mice.

In this study, we report characterization of the gene Tmtc4 (transmembrane and tetratricopeptide repeat 4 ), which when genetically inactivated in mice, causes rapid and early postnatal outer and inner cochlear hair cell death, leading to hearing loss. We demonstrate that the protein encoded by Tmtc4 can be found in the same molecular complex with the main endoplasmic reticulum (ER) $\mathrm{Ca}^{2+}$ pump, SERCA2b, and through this may alter $\mathrm{ER} \mathrm{Ca}^{2+}$ dynamics, leading to downstream overactivation of the ER unfolded protein response (UPR) and cell death. We confirm the functional linkage between Tmtc4 and the UPR by conducting a genetic "inverse complementation" experiment, in which combined genetic inactivation of both Tmtc4 and the ER stress constituent Chop leads to reduced hearing loss when compared with inactivation of Tmtc4 alone. We then demonstrate that these mechanisms are also altered early in the cochlea's response to acoustic overstimulation and that pharma- 
A

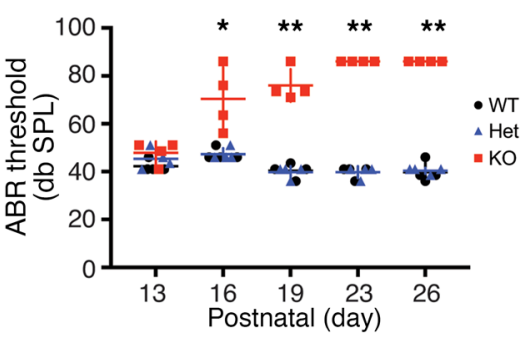

B

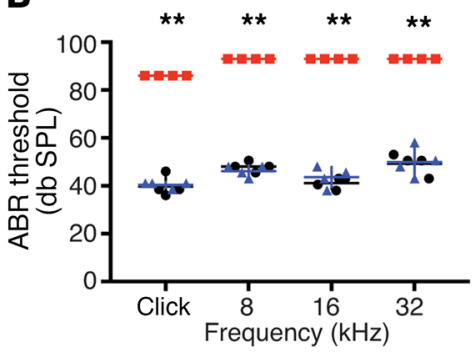

D

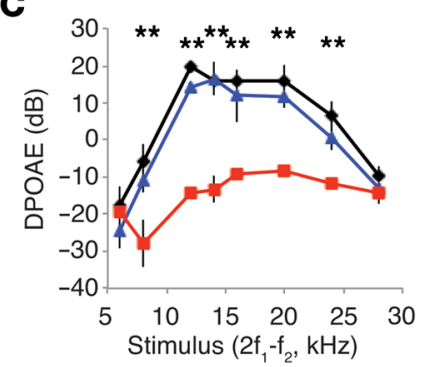

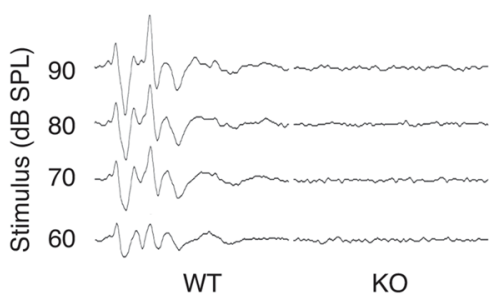

Figure 1. Rapidly progressive postnatal hearing loss in Tmtc4-KO mice. (A) At postnatal day 13, auditory brainstem response (ABR) thresholds to broadband click stimuli are equivalent in Tmtc4-KO (red squares), Het (blue triangles), and WT (black circles) littermates. Tmtc4-KO mice progress to profound deafness by P26 ( $n$ = 4 for each genotype). (B) Hearing loss in P26 Tmtc4-KO mice is present in response to both broadband click and a range of pure-tone frequencies ( $n=4$ for each genotype). (c) Distortion-product otoacoustic emissions (DPOAEs) measured at different frequencies demonstrate cochlear dysfunction in P26 Tmtc4-KO mice ( $n=4$ for each genotype). (D) ABR waveforms in P26 Tmtc4-KO and WT littermates demonstrate absent $A B R$ responses to click stimuli at multiple sound pressure levels (SPL) in KO mice. Traces representative of at least 4 experiments. Data are mean \pm SD. ${ }^{*} P<0.01,{ }^{*} P<0.001$ by 2 -tailed, unpaired $t$ test. cological inhibition of this ER stress response rescues a mouse model of NIHL, pointing to both novel mechanisms of and novel treatments for hearing loss.

\section{Results}

Tmtc4-knockout mice have rapid postnatal-onset hearing loss. ES cells were obtained from the knockout (KO) mouse project repository (www.komp.org) that contained a nonconditional genomic deletion within Tmtc4 via homologous recombination (15) on a C57BL/6J background. This deletion begins at the $5^{\prime}$ end of exon 1 through the $3^{\prime}$ end of exon 3 , replaced by a cassette encoding the genes for $\beta$-galactosidase ( $\beta$-gal) and neomycin (Supplemental Figure 1A; supplemental material available online with this article; https://doi.org/10.1172/JCI97498DS1). After confirming that these ES cells carried the Tmtc4 interstitial deletion, these cells were then injected into the blastocysts of C57BL/6J (B6) mice. Vertical transmission was confirmed and $\mathrm{KO}$ and heterozygous (Het) offspring were viable, and $\mathrm{KO} / \mathrm{WT} /$ Het mice were born with the predicted Mendelian frequencies. There was no evident morbidity or early mortality, and the $\mathrm{KO}$ animals reproduced successfully.

Tmtc4-KO mice have early onset hearing loss when compared with their matched littermate controls. Sound-field click and pure-tone auditory brainstem response (ABR) showed that hearing thresholds measured at P13 were identical among WT, $\mathrm{KO}$, and Het mice (Figure 1A). However, by P23 the KO mice were nearly completely deaf (Figure 1, A, B, and D). Distortionproduct otoacoustic emissions (DPOAEs) were also absent, indicating outer hair cell (OHC) dysfunction (Figure 1C). Immunohistochemistry of cochlear explants revealed progressive loss of first OHCs and then inner hair cells (IHCs) at the cochlear base in Tmtc4-KO mice compared with WT littermates (Figure 2, A, B, D, and E), and subsequently at the mid-turn and apex (data not shown). Histologic analysis corroborated this finding of generalized and progressive cochlear degeneration affecting first hair cells, and subsequently the supporting cell network and spiral ganglion neurons (Figure 2, C and F, and Supplemental
Figure 2). To provide further evidence that inactivation of Tmtc4 is the cause of hearing loss in these mice, we backcrossed the Tmtc4 mutant allele (that was initially on the B6 background) for 10 generations (N10) onto the FVB background, a strain well established for normal hearing thresholds, unlike the B6 mice on which the KO was initially generated (which carries the hearing loss ahl allele; ref. 16). These FVB N10 $\mathrm{Tmtc4}^{+/-}$mice, when intercrossed to produce $\mathrm{Tmtc} 4^{-/-}$mice, produced viable and healthy offspring of the expected Mendelian ratios. Moreover, as seen in the original $\mathrm{B} 6$ line, when ABR thresholds were measured at P23, the KO mice were deaf (ABR waveforms not detected at $85 \mathrm{~dB}$ ) and the WT and Het mice had normal thresholds (data not shown), providing additional evidence that inactivation of Tmtc 4 is the cause of hearing loss.

Tmtc 4 is broadly expressed in the cochlea and enriched in the endoplasmic reticulum. Immunohistochemistry on cochlear sections from P21 Tmtc4 Het mice demonstrated broad expression of $\beta$-gal (as an indirect measure of Tmtc4 expression) driven by the Tmtc4 promoter in IHCs, OHCs, and cochlear supporting cells (Figure 3). This corroborates recently published data from RNASeq and mass spectrometry experiments of flow-sorted outer and inner hair cells confirming that Tmtc 4 protein is highly enriched in these cells (17, 18). These published data also demonstrate that the level of Tmtc4 (both mRNA and protein) in the cochlea is higher than the other 3 isoforms (Tmtc1,2,3), although all 4 are expressed in the cochlea (18). Given the broad expression of all isoforms in the cochlea and the recently published linkage of TMTC2 to hearing loss in humans (19), we also tested whether inactivation of Tmtc4 led to a change in the abundance of other isoforms. As measured by quantitative PCR (qPCR), we found that there was a significant increase in the levels of Tmtc1, Tmtc2, and Tmtc3 mRNA in the cochlea of Tmtc4KO mice compared with littermate WT controls, suggesting possible shared functions (Supplemental Figure 3).

We then assessed the intracellular localization of TMTC4. We stably introduced c-Myc-tagged TMTC4 cDNA into HEK 293 cells and assessed intracellular localization of TMTC4-Myc using anti- 

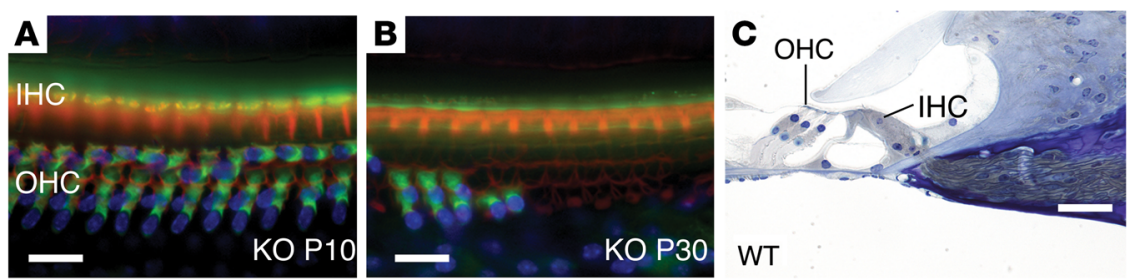

Figure 2. Progressive hair cell loss in Tmtc4-KO mice. Staining for actin (red; phalloidin), myosin7a (green), and nuclei (blue; DAPI) demonstrates progressive loss of outer hair cells $(\mathrm{OHC})$ and inner hair cells (IHC) in the cochlear base from Tmtc4-KO mice at P10 (A), P30 (B), and P45 (D). Cochlea from a P30 WT mouse is shown for comparison (E). Histologic sections from P26 organ of Corti in WT (C) and KO (F)
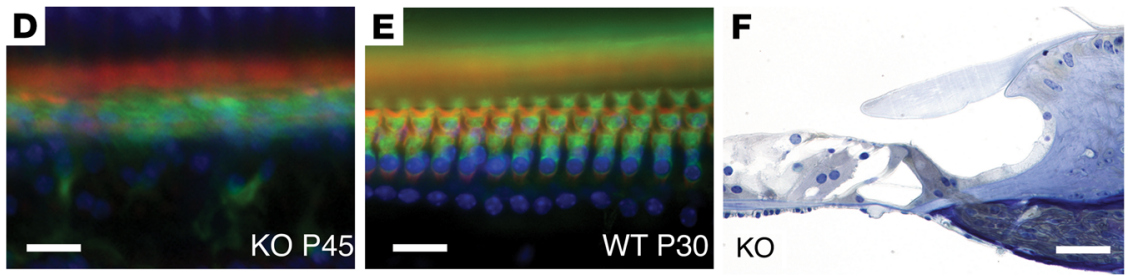
mice show disrupted architecture in the KO cochlea. Images representative of at least 4 experiments each. Scale bar: $10 \mu \mathrm{m}$ in A, B, D, and E. Scale bar: $50 \mu \mathrm{m}$ in $\mathbf{C}$ and $\mathbf{F}$.

body against human TMTC4 (Figure 4A). This approach showed overlap of localization of TMTC4 with the ER-resident proteins SERCA2B and GRP94 (89\% and 87\%, respectively), but minimal overlap was noted with staining of the Golgi marker GM130 (29\%), suggesting that TMTC4 was enriched in the ER. We then tested whether native TMTC4 was overrepresented in biochemical fractions enriched in the endoplasmic reticulum from untransfected, WT HEK cells. We found, as would be predicted, that SERCA2B, calnexin, and calreticulin were enriched in the ER fraction, but VDAC1, a mitochondrial marker, was not. TMTC4 was found to be enriched in the same cellular fraction as the ER markers (Figure 4B). To further test whether TMTC4 may be an ER-resident protein, we immunoisolated TMTC4-Myc from stably transfected HEK cells and then treated this fusion protein with endoglycosidase $\mathrm{H}$ (Endo $\mathrm{H}$ ) or peptide $\mathrm{N}$-glycosidase $\mathrm{F}$ (PNGase F). N-linked glycans are large hydrophilic sugar structures that are added co- or posttranslationally to most secretory proteins upon ER targeting. TMTC4 is predicted to have multiple glycan sites by the NetNGly algorithm (20). As shown in Figure $4 \mathrm{C}$, both Endo $\mathrm{H}$ and PNGase F lead to a decrease in the apparent molecular weight of TMTC4-Myc, further supporting the hypothesis that TMTC4 is a glycosylated ER-resident protein.

Next, we examined the potential interaction partners of TMTC4 in human fetal brain and in HEK cells stably transfected with TMTC4-Myc (Figure 5). From nonnuclear detergent solubilized whole-cell lysate, 2 known ER markers - SERCA2B and calnexin - were identified to coimmunoprecipitate with either endogenous TMTC4 from human brain (Figure 5B) or stably transfected TMTC4-Myc (Figure 5A) from human HEK293 cells by using an antibody against human TMTC4. These complementary approaches suggest that TMTC4 is a resident ER membrane protein that complexes with SERCA2B and calnexin, 2 ER membrane proteins that mediate $\mathrm{Ca}^{2+}$ and UPR dynamics.

Tmtc4 regulates $E R \mathrm{Ca}^{2+}$ dynamics. The data above suggest that TMTC4 binds to a protein complex that includes the principal $\mathrm{Ca}^{2+}$ pump in the ER, SERCA2B, which, by pumping $\mathrm{Ca}^{2+}$ into the ER, helps to maintain the approximately 5,000-fold $\mathrm{Ca}^{2+}$ gradient between the ER and the cytoplasm (21). We thus sought to test the role of Tmtc 4 in $\mathrm{ER} \mathrm{Ca}^{2+}$ dynamics. We measured intracellular cytosolic $\left[\mathrm{Ca}^{2+}\right]$ in WT and Tmtc4-KO cochleae using live FURA-2 ratiometric $\mathrm{Ca}^{2+}$ imaging (22). In neonatal cochlear organotypic cultures, spontaneous intercellular $\mathrm{Ca}^{2+}$ waves occur between the supporting cells of the organ of Corti (Figure 6A). Within individual cells, peaks correspond to cytosolic $\mathrm{Ca}^{2+}$ increase due to release of $\mathrm{Ca}^{2+}$ from intracellular ER stores, followed by a return to baseline, due in part to subsequent $\mathrm{Ca}^{2+}$ reuptake by SERCA2b back into the ER (23). Quantification of the characteristics of these $\mathrm{Ca}^{2+}$ peaks, in particular, the decay time from the maximal cytosolic $\left[\mathrm{Ca}^{2+}\right]$ halfway back to the baseline, provides insight into $\mathrm{ER} \mathrm{Ca}^{2+}$ reuptake kinetics. In both neonatal and adult cochleae, these $\mathrm{Ca}^{2+}$ waves are thought to be involved in the cochlear response to mechanical and acoustic trauma (22, 24). Thus, we used this model to investigate the role of Tmtc 4 in $\mathrm{ER} \mathrm{Ca}^{2+}$ reuptake.

Application of $1 \mu \mathrm{M}$ thapsigargin, an irreversible inhibitor of SERCA2b, which leads to depletion of $\mathrm{Ca}^{2+}$ in the ER, induction of the UPR, and cell death (25), and $10 \mu \mathrm{M}$ CDN1163, a SERCA2b positive allosteric modulator (26), respectively increase and decrease this decay time in WT cochlear cultures (Figure 6, B and F), demonstrating that $\mathrm{Ca}^{2+}$ peak decay time can serve as a proxy for measurement of $\mathrm{ER} \mathrm{Ca}^{2+}$ reuptake. Tmtc4-KO cochleae had significantly increased spontaneous $\mathrm{Ca}^{2+}$ wave frequency and higher $\left[\mathrm{Ca}^{2+}\right]_{i}$ peak levels (Figure 6, D and E). Most notably, similar to thapsigargin, Tmtc4-KO cochlea had significantly longer decay time of $\left[\mathrm{Ca}^{2+}\right]$ return to baseline (Figure 6, $\mathrm{C}$ and $\mathrm{F}$ ). These findings together are all consistent with impaired $\mathrm{Ca}^{2+}$ reuptake into the ER in Tmtc4-KO cochleae, and suggest that Tmtc4 is a necessary component for regulating $\mathrm{ER} \mathrm{Ca}^{2+}$ levels.

TMTC4 participates in ER stress and the UPR. The aforementioned experiments demonstrated that Tmtc4-KO mice exhibit early postnatal hearing loss and corresponding progressive degeneration of OHCs, IHCs, and supporting cells. We showed that Tmtc4 is an ER resident protein, associates with the $\mathrm{Ca}^{2+}$ reuptake pump SERCA2b, and is involved in $\mathrm{ER} \mathrm{Ca}^{2+}$ dynamics. Dysregulation of $\mathrm{ER} \mathrm{Ca}^{2+}$ is strongly associated with ER stress and the UPR pathway (27). We hypothesized that the observed hearing loss in Tmtc4-KO mice may be a result of aberrant overactivation of the UPR and subsequent cell death.

To test the role of Tmtc4 in the UPR, we isolated fibroblasts from the skin of newborn mice (WT and KO) and tested for activation of key signaling molecules in the UPR. WT and KO fibroblasts were treated for 2 hours with $1 \mu \mathrm{M}$ thapsigargin. KO cells showed increased activation of all 3 arms of the UPR with markers selective for each arm: Chop (reflective of the PERK arm), 

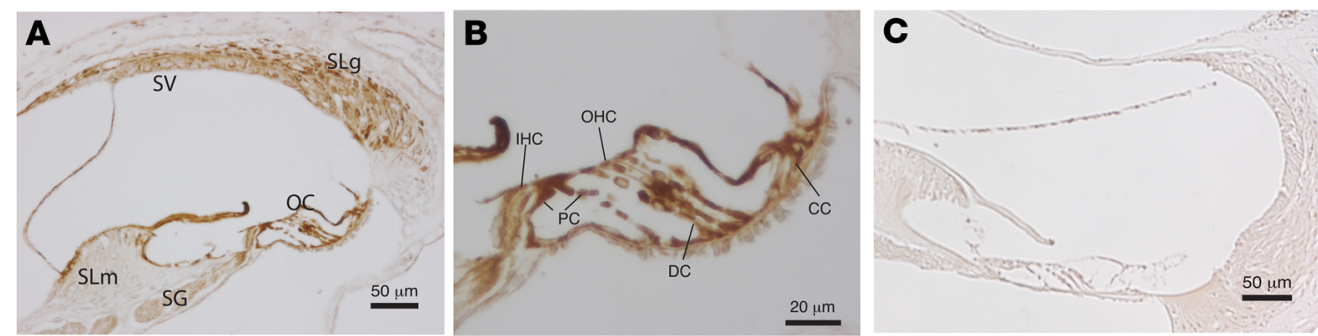

Figure 3. Expression of Tmtc4 and its isoforms in mice. In heterozygous Tmtc4-KO mice, $\beta$-gal expression is driven in the KO allele by the Tmtc4 promoter. $\beta$-gal expression is seen broadly in the cochlea, particularly in the stria vascularis (SV), spiral ligament (SLg), and organ of Corti (OC), with less expression noted in the spiral limbus (SLm) and spiral ganglion (SC). (B) In a magnified view of A, the organ of Corti contains inner (IHC) and outer (OHC) hair cells, as well as supporting cells (pillar cells [PC], Dieter's cells [DC], and Claudius cells [CC]), with robust labelling. (C) WT control mice, in which Tmtc4 promoterdriven $\beta$-gal expression is not present, shows no $\beta$-gal antibody labeling, demonstrating that the broad labelling seen in $\mathbf{A}$ and $\mathbf{B}$ is specific.

XBP1 (reflective of the IRE1 arm), and BiP (Figure 7A). Fluorescence-activated cell sorting showed that steady-state Chop protein levels (as a surrogate for the arm of the UPR that more consistently promotes cell death) are markedly higher in KO compared with WT fibroblasts, with $29.9 \%$ (KO) versus $0.5 \%$ (WT) of cells having expression levels above threshold, indicating overactivation of the UPR in KO cells even at baseline in the absence of thapsigargin treatment (Figure 7B). These findings were further confirmed by Western blotting of cells treated with thapsigargin for 24 hours. Compared with WT fibroblasts, Tmtc4-KO fibroblasts had significantly higher protein levels of Chop as well as the downstream UPR protein DR5, and the apoptosis marker cleaved caspase 8 (Supplemental Figure 4 and ref. 28).

We then directly tested whether the UPR was overactivated in cochleae from KO versus WT mice. Neonatal cochlear cultures were treated with $1 \mu \mathrm{M}$ thapsigargin or with vehicle for 2 hours. As was seen in cultured fibroblasts, KO cochleae had greater induction of mRNA for UPR activators compared with WT cochleae (Figure 7C). Additionally, caspase 3 mRNA was found to be elevated only in thapsigargin-treated KO cochleae, demonstrating relative sensitivity to apoptotic cell death in $\mathrm{KO}$, but not WT cochlea subjected to pharmacologic ER stress in this model (Figure 7C). These data together demonstrate that absence of Tmtc4 leads to overactivation of the UPR and cell death, both in KO fibroblasts and in Tmtc4-KO neonatal cochleae, suggesting that this cellular disruption leads to hearing loss in these mice.

Noise exposure activates the UPR in WT mice. We hypothesized that activation of the UPR may be a more general mechanism of hearing loss and tested directly whether acoustic overstimulation (as a model for NIHL) results in UPR activation in WT mice. Eight-week-old WT FVB mice were exposed to $8-16 \mathrm{kHz}$ octaveband white noise at a $106 \mathrm{~dB}$ sound pressure level (SPL) for 120 minutes to generate a transient threshold shift with partial recovery and partial permanent threshold shift (22). UPR pathway gene expression was upregulated as measured in the dissected cochleae 2 hours after completion of noise exposure compared with unexposed mice (Figure 8A). These findings strongly implicate UPR upregulation in the early stages of NIHL. Additionally, caspase 3 mRNA levels are also elevated in mice exposed to loud noise, further strengthening this association between the UPR, downstream cell death, and NIHL (Figure 8A).
Modulating the UPR prevents hearing loss in both environmental and genetic models. Given this observed link of the UPR to hearing loss in both genetic and environmental models, we hypothesized that modulating the UPR could protect against hearing loss. Of the 3 arms of the UPR, the one culminating in the upregulation of Chop is most consistently implicated in promoting apoptotic cell death, and might be a good candidate for pharmacological and genetic modulation (27). ISRIB (integrated stress response inhibitor) binds to and activates the guanine nucleotide exchange factor (GEF) eIF2B, leading to subsequent inhibition of ATF4 and hence suppression of downstream components of the UPR, including Chop $(29,30)$. We first assessed whether ISRIB can modulate the UPR in our cell-based models. After $1 \mu \mathrm{M}$ thapsigargin treatment to induce UPR activation, WT fibroblasts treated with ISRIB showed decreased levels of both CHOP and S-XBP1 compared with fibroblasts treated with thapsigargin alone (Supplemental Figure 5). We then evaluated the ability of ISRIB to attenuate NIHL. WT FVB mice were exposed to the same sound described above, which we showed induces UPR gene upregulation (Figure $8 \mathrm{~A})$. ISRIB $(2.5 \mathrm{mg} / \mathrm{kg})$ or vehicle was administered intraperitoneally 3 hours prior to noise exposure. ABR thresholds 1,7 , and 14 days after noise exposure were elevated in both groups of animals, but those treated with ISRIB showed improved hearing at all time points, with approximately $30 \%$ attenuation of threshold shifts ( $P<0.05$ for each time point) (Figure $8 \mathrm{~B})$. In addition to this partial rescue of hearing loss, we also observed a corresponding decrease in outer hair cell loss in these same ISRIB-treated and noise-exposed mice (Figure 8, C and D).

These findings demonstrated the ability of UPR modulation, specifically inhibition of the Chop arm of the UPR, to prevent hearing loss in an acquired, pharmacologic model (Figure 9A). We next performed a genetic "inverse complementation" assay by breeding Tmtc4-KO mice to Ddit3 KO (Chop-KO) mice. A previous study has shown that Chop-KO mice were less susceptible to the deleterious effects of aberrant UPR regulation, providing a strong rationale for this approach (refs. 31-33 and Figure 9B). Compared with Tmtc4-KO mice on a Chop WT background, animals that were homozygous for both the Tmtc4-KO and the Chop-KO alleles had improved hearing thresholds at P19, although not completely restored, as this may be a result of the more significant impact on the UPR that complete absence of Tmtc 4 creates (Figure 9C). 
A
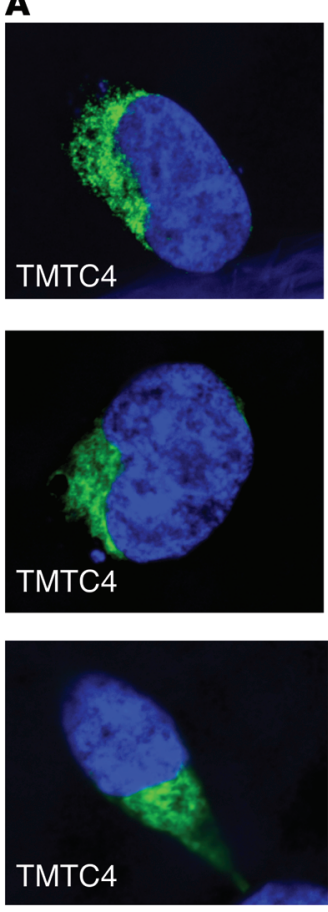

GRP94
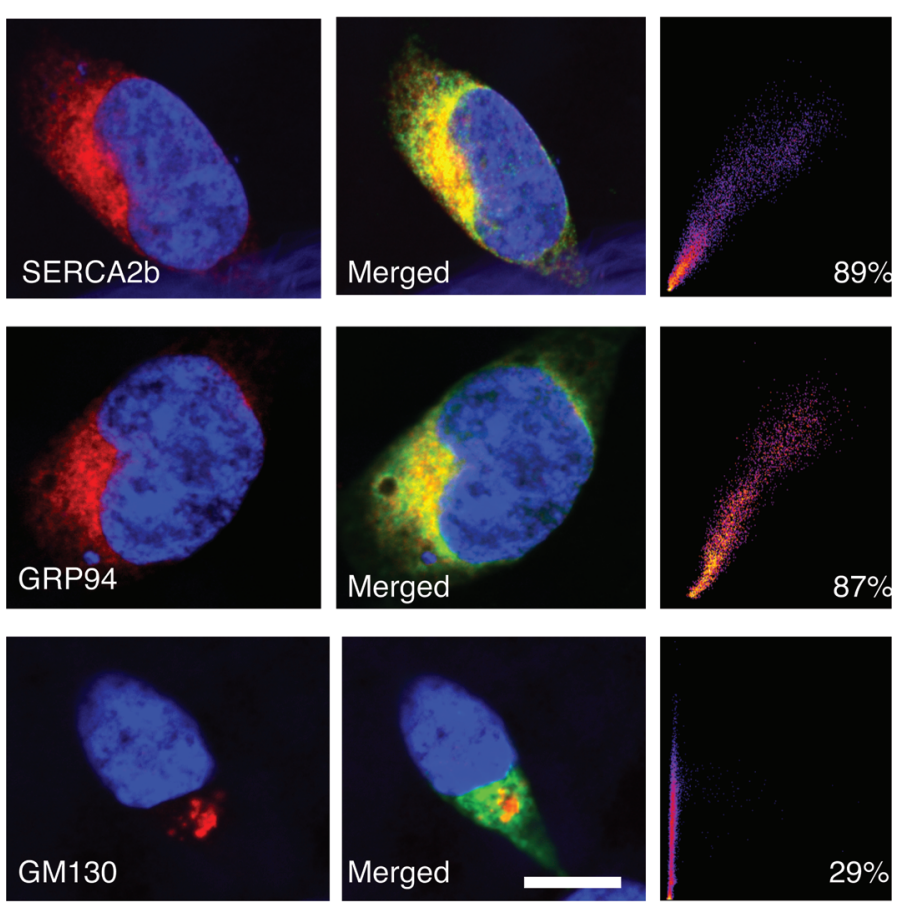

B
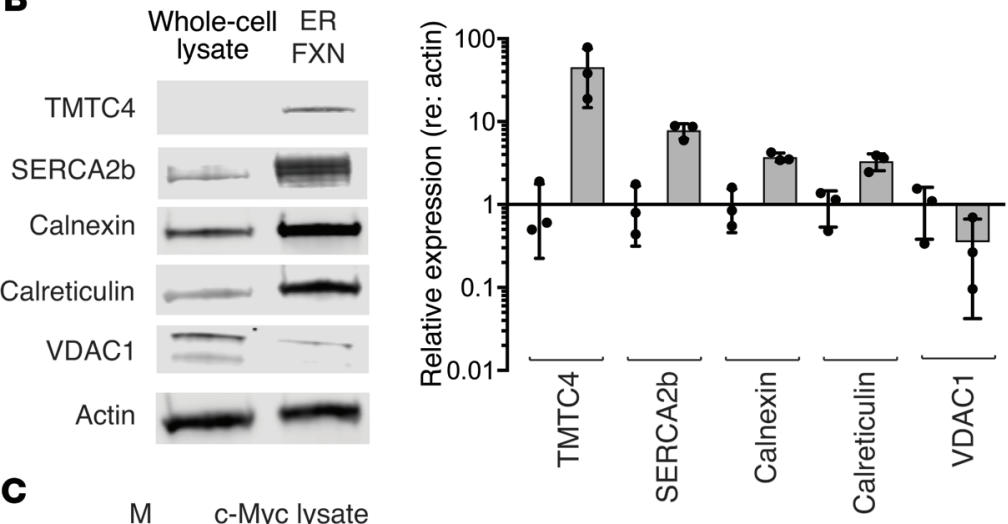

Figure 4. TMTC4 enrichment in the ER and colocalization with ER markers. (A) HEK cells were stably transfected with TMTC4-c-myc constructs and stained with antibodies against TMTC4 (green, first column), ER proteins (CRP94 or SERCA2b), or the Golgi-enriched protein GM130 (red, second column), and DAPI to stain nuclei (blue, all panels). As demonstrated in the merged panels (third column), TMTC4 colocalizes with ER proteins but not with the Golgi protein (quantified in the fourth column). Images are representative of 5 experiments. Scale bar: $10 \mu \mathrm{m}$. (B) Nonnuclear whole-cell lysate (whole-cell lysate) from WT HEK cells, and a fraction enriched for ER membranes (ER Fxn), were stained with antibodies against TMTC4, as well as to the known ER resident proteins SERCA2b, calnexin, and calreticulin; all showed enrichment in the ER. As controls, actin was not enriched, and the mitochondrial membrane protein VDAC1 was barely detected, in the ER fraction. Quantification of expression levels (relative to actin, and normalized against the relative expression level in whole-cell lysate) confirm the ER localization of endogenous TMTC4 $(n=3$ experiments, from which representative images were taken). (c) TMTC4-c-Myc and copurifying proteins were isolated from a postnuclear supernatant of HEK cells with stably incorporated TMTC4-c-Myc vector. This lysate (c-Myc lysate) was then treated with or without Endo $\mathrm{H}$ and/or PNGase $\mathrm{F}$ and the fractions assessed with antibody against c-Myc. The shift in molecular weight in TMTC4 treated with both Endo $\mathrm{H}$ and PNGase $\mathrm{F}$ indicates that TMTC4 is glycosylated, consistent with residence in the ER. M: molecular weight markers. Images are representative of 3 experiments.
C

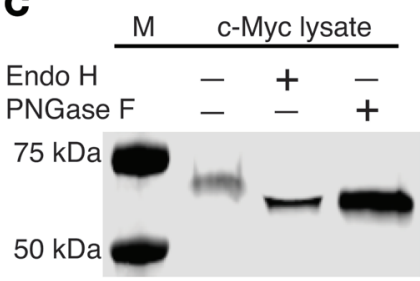

This suggests that the Chop-KO allele complements the absence of Tmtc 4 and decreases the severity of hearing loss, linking these 2 genes to a shared molecular pathway, and underscoring the importance of the UPR as a potential pathway for treatment for NIHL.

\section{Discussion}

We have shown that the gene Tmtc4, when inactivated, leads to rapid onset postnatal hearing loss with subsequent hair cell death and cochlear degeneration. We demonstrated that inactivation of Tmtc4 in the mouse cochlea leads to aberrant $\mathrm{Ca}^{2+}$ signaling, consistent with delayed reuptake into the ER, and to overactivation of the UPR, as observed by an increase in MRNA and protein levels for key UPR proteins Chop, XBP1, and BiP, and apoptotic proteins DR5 and caspase 8 . Our data show that Tmtc4 is present in cochlear supporting and hair cells, is enriched in the ER, and coimmunoprecipitates with SERCA2b, which strengthens the linkage between the observed aberrant regulation of $\mathrm{ER} \mathrm{Ca}^{2+}$ and Tmtc4. By use of an inverse genetic complementation approach, we confirmed the importance of the UPR in mediating hearing loss in this genetic model, showing that simultaneous homozygous inactivation of both Tmtc4 and Chop leads to less impaired hearing than with Tmtc4-KO alone. We extended these important advances underlying the genetics and biology of hearing loss by demonstrating for what we believe is the first time that NIHL is associated with activation of the UPR, which points to new avenues for treatment for this common disabling condition. 
A

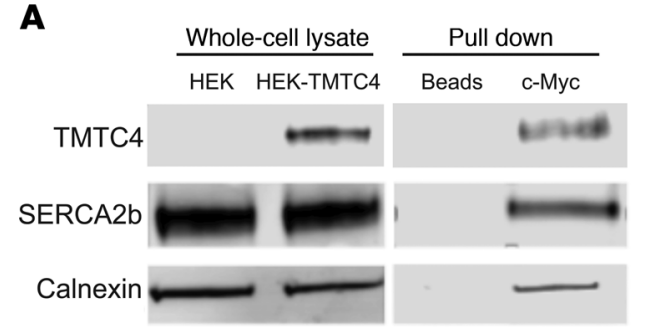

B

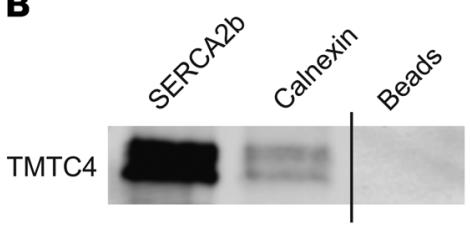

Figure 5. TMTC4 coimmunoprecipitation with ER membrane proteins. (A) Nonnuclear wholecell lysates from HEK cells with stably incorporated TMTC4-c-Myc vector (HEK-TMTC4) or empty plasmid (HEK) were assayed with antibodies against ER proteins (SERCA2b, calnexin) or TMTC4 showing that all 3 proteins were present in transfected HEK cells and that the TMTC4 antibody did not stain nonspecifically in mock-transfected cells. These extracts from TMTC4-c-Myc stably transfected cells were then incubated with agarose beads linked with antibodies against c-Myc or to beads alone (Beads). Bound proteins were purified and the "pull down" fraction stained with the aforementioned antibodies against TMTC4 and ER proteins, showing that SERCA2b and calnexin are copurified upon c-Myc pull down. Images are representative of 3 experiments. (B) Human fetal brain lysate was used to assess endogenous TMTC4 interactions. Pull down fractions were isolated using beads linked to antibody against SERCA2b, calnexin, or beads alone without linked antibodies (Beads), and probed with antibody against TMTC4. Endogenous TMTC4 was found to coimmunoprecipitate strongly with SERCA2b and weakly with calnexin. Images are representative of 3 experiments.

We tested this therapeutic hypothesis directly by showing that pharmacological suppression of the UPR with a single dose of the eIF2B-activating (and indirectly ATF4-inhibiting) drug ISRIB considerably lessens hearing loss caused by noise exposure. These compelling findings, through both pharmacological and genetic approaches to treat hearing loss, provide new insights into the mechanisms of hearing impairment and suggest a highly promising target for treatment of one of the most common causes of hearing loss.

Tmtc4 is a gene encoding a predicted 741 amino acid $83 \mathrm{kDa}$ protein. Tmtc 4 has been found by both proteomic and transcriptomic analyses to be expressed in the developing and young postnatal murine cochlea along with the other 3 isoforms, which is consistent with our data $(17,18,34)$. Moreover, our finding that $T m t c 1, T m t c 2$, and Tmtc3 all increase substantially in transcript abundance in cochleae from Tmtc4-KO mice appears to underscore the likely interrelatedness of these genes. This connection may extend to hearing loss in patients as well. In parallel with our work recently, a missense mutation (rs35725509; Val136Ile) in the human ortholog TMTC2 was linked to nonsyndromic sensorineural hearing loss both in a 6-generation family and in a cohort of unrelated individuals with SNHL (179 affected vs. 186 matched controls) $(19,35)$. This mutation was also recently identified in another family dyad with hearing loss (35). Inactivation of Tmtc4 in a recently published large-scale genomics manuscript concurrent with our work was confirmed by a separate group to result in hearing loss, linking this class of proteins more strongly to hearing impairment (36). TMTC4 encodes 10 putative transmembrane domains and 8 tetratricopeptide repeat (TPR) domains (Supplemental Figure 1D). TPR domains have been implicated in the generation and regulation of many multiprotein complexes in cellular processes such as transcription and protein degradation. The UPR chaperone protein P58(IPK) also has 9 TPR repeat motifs, and these repeat domains are necessary for the protein's chaperone activity (37). Thus, by comparison, it is possible that the TPR repeats in TMTC4 may sense unfolded proteins and regulate $\mathrm{Ca}^{2+}$ signaling through this mechanism. TMTC4 also contains an ER retention signal, which may help locate the protein to the ER, and hence to regulate $\mathrm{Ca}^{2+}$ flux and the unfolded protein response. The TMTC4 protein is highly conserved from mammals to fish and invertebrates, but is not present in plants, fungi, or bacteria. TMTC4 is 1 of 4 genes in this family, diverging evolutionarily from the paralogs TMTC1-3 approximately 1.2 billion years ago. Despite this more distant divergence, there is still evidence of potential shared functions. Like TMTC4, both TMTC1 and TMTC2 are enriched in the ER and coimmunoprecipitate with SERCA2B (38). There is preliminary large-scale evidence from genome-wide association study linking TMTC1, TMTC2, and TMTC4 to type 2 diabetes, a disease whose pathogenesis has been linked strongly to ER stress and the UPR (39, 40). Separately, a recent study suggested that the Tmtc class of proteins may also function as components of an O-mannosylation pathway that selectively glycosylates cadherins and protocadherins, many of which, especially Cdh23, play critical roles in hair cell function and have also been tied to ER stress and the UPR $(41,42)$. Further study into the precise role of TMTC family members, and particularly TMTC4, in O-mannosylation, $\mathrm{ER} \mathrm{Ca}^{2+}$ flux, and the UPR in the cochlea would yield valuable insight into this important pathway.

The UPR is an essential component of cell homeostasis. In response to such stressors as excessive protein synthesis, this UPR pathway can be activated in a manner that slows protein synthesis and promotes cell survival $(43,44)$. However, if the UPR is overactivated, this aberrant signaling can lead to cell death. There is precedence for involvement of the UPR in genetically defined disorders associated with hearing loss. One disorder, Wolfram syndrome, presents clinically with diabetes insipidus, diabetes mellitus, optic atrophy, and deafness (also referred to as DIDMOAD syndrome). One of the genes for this disorder, WFS1, has been implicated in ER stress through multiple mechanisms, including binding to and regulating the ER $\mathrm{Ca}^{2+}$ pump SERCA2B (45). Thus, this disorder links diabetes, deafness, and neuronal cell loss to ER stress. A related disorder, Darier-White disease (DWD), is caused by autosomal dominant mutations in the gene ATP2A2, which encodes the same SERCA2B ER Ca ${ }^{2+}$ pump $(46,47)$. In the general population, this protein is highly intolerant of loss-of-function mutations as assessed by data from the exome aggregation consortium (EXAC. broadinstitute.org), and there are no reports of patients with homozygous mutations. DWD presents typically with painful mucosal and skin lesions (46) and sometimes deafness, further strengthening the linkage that we observe with TMTC4 (47). While these associations show more direct linkage of the UPR to hearing loss, there are also examples where indirect activation of ER stress occurs in deafness syndromes. For example, in Usher syndrome, which can be caused by mutations in proteins encoding Cdh23, harmonin, 

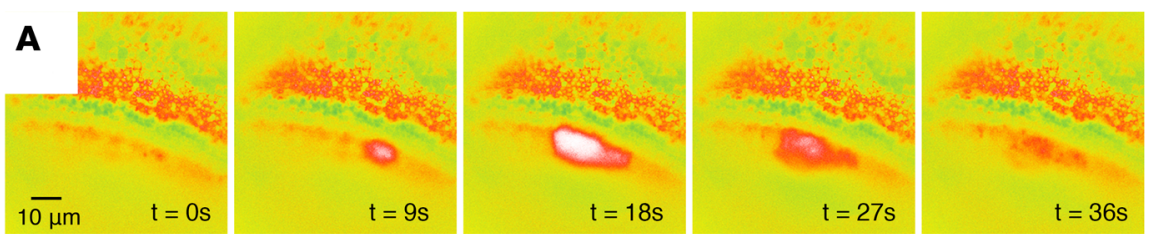

B

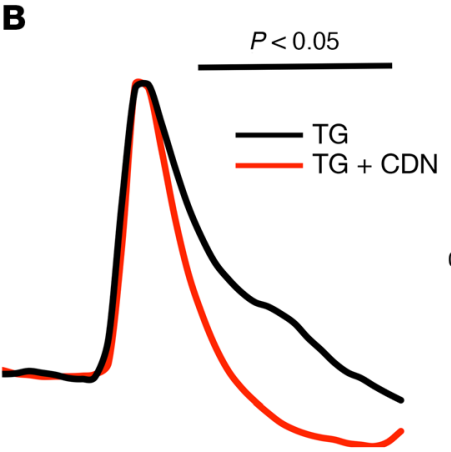

0.2

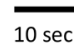

C

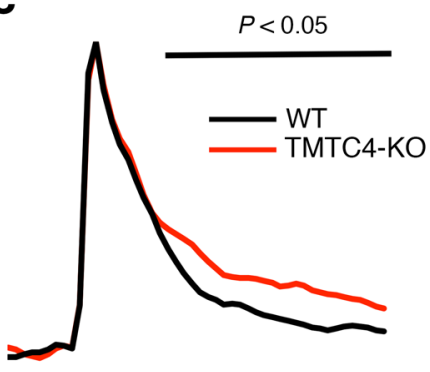

D

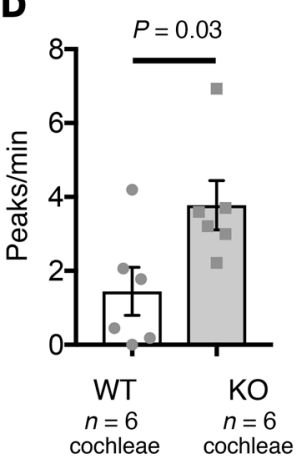

$\mathbf{E}$

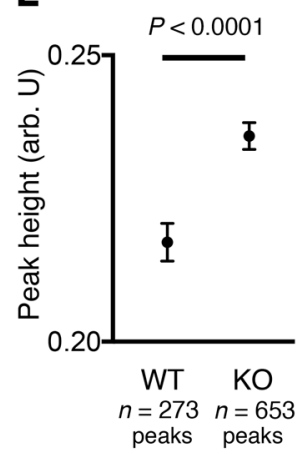

\section{$\mathbf{F}$}

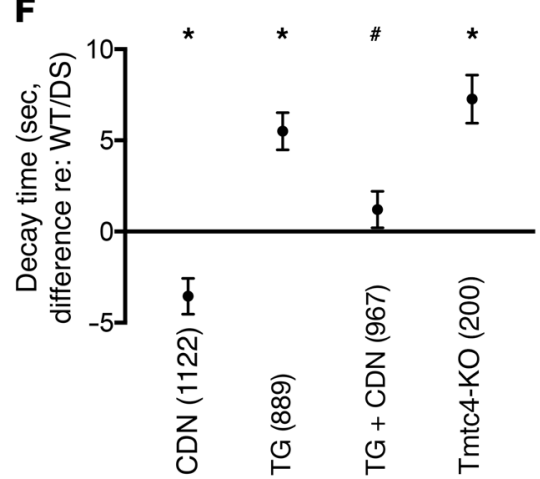

Figure 6. $\mathrm{Ca}^{2+}$ dynamics in cochlear supporting cells. Ratiometric FURA-2 imaging was used to measure cytosolic $\left[\mathrm{Ca}^{2+}\right]_{\mathrm{i}}$ in WT and Tmtc4-KO P3-P5 cochlear cultures. (A) Representative spontaneous intracellular $\mathrm{Ca}^{2+}$ wave in the inner sulcus of the organ of Corti. Regions of interest (7- $\mu \mathrm{m}$ square) in the inner and outer sulci were identified and FURA-2 excitation ratios (proportional to cytosolic $\mathrm{Ca}^{2+}$ concentration) measured. $\mathrm{Ca}^{2+}$ peaks were extracted and analyzed. (B) Aggregate ratio time course for $\mathrm{Ca}^{2+}$ peaks normalized to baseline and peak maximum show identical initial kinetics and faster return to baseline in WT cochleae treated with the SERCA2B activator CDN1163 (CDN) plus the SERCA2B inhibitor thapsigargin (TC) ( $n=88$ peaks) compared with TC ( $n=65$ peaks) alone, demonstrating the ability of known modulators of ER $\mathrm{Ca}^{2+}$ reuptake to affect $\mathrm{Ca}^{2+}$ peak decay time. (C-E) Analysis of 273 and 653 peaks, respectively, from 6 WT and 6 KO cochleae. (C) Peaks in Tmtc4-KO cochleae ( $n=653$ ) showed prolongation of return to baseline cytosolic $\mathrm{Ca}^{2+}$ level relative to WT controls $(n=273)$. (B and C) Black bars: points at which aggregate ratios were statistically significantly different. Peak frequency (D) (mean \pm SEM) and height $(\mathbf{E})$ (mean $\pm \mathrm{SEM}$ ) were greater in $\mathrm{KO}$ cochleae. (F) Decay time in WT cochleae was measured in dissecting solution (DS) and with drugs (CDN: $10 \mu \mathrm{M}$ CDN1163; TC: $1 \mu \mathrm{M}$ TC; and TG+CDN: $10 \mu \mathrm{M}$ CDN1163 and $1 \mu \mathrm{M}$ TC) as well as for Tmtc4-KO cochleae in DS. Data indicate difference in mean $\pm \mathrm{SE}$ of decay time relative to that measured in WT cochleae in DS (number of peaks analyzed for each comparison in parentheses). ${ }^{*} P<0.01$ for DS in WT cochleae. ${ }^{\#} P<0.0001$ versus TC in WT cochleae (unpaired, 2-tailed $t$ test for all comparisons). and Myo7a, mutated isoforms of these genes prevent the preassembly of this plasma membrane protein complex in the ER, leading to activation of ER stress and subsequent apoptosis (42). Thus, these examples provide both indirect and direct evidence linking ER stress and the UPR to hearing loss. Moreover, there is also data linking age-related hearing loss to the $\operatorname{UPR}(17,48)$.

Our work with TMTC4 further strengthens the connection between the UPR and hearing loss, but also implicates the even more upstream pathway of $\mathrm{Ca}^{2+}$ regulation, which may be a potentially more advantageous point for therapeutic intervention. Activation of intercellular $\mathrm{Ca}^{2+}$ signaling (ICS) waves in cochlear supporting cells, which are dependent upon $\mathrm{Ca}^{2+}$ flux into and out of the ER, have been implicated as an early mechanism in cochlear trauma, analogous to their well-studied role in neurodegeneration. In both neonatal cochlear cultures and, more recently, mature-hearing cochlear explants, ICS waves are triggered within minutes of mechanical and acoustic trauma $(22,24)$. In this study, we have found that properties of these ICS waves are altered in Tmtc4-deficient mice in ways consistent with impaired $\mathrm{Ca}^{2+}$ reuptake: cytosolic $\mathrm{Ca}^{2+}$ clearance is delayed and ICS waves are more frequent. Modulation of $\mathrm{ER} \mathrm{Ca}^{2+}$ dynamics, therefore, may be a promising avenue for hearing loss therapeutics, as has been shown recently in other UPR-dependent disease models; for example, an allosteric activator of SERCA2b, CDN1163, attenuates the development of insulin resistance (type 2 diabetes) in the Ob/Ob mouse (26). Current efforts to treat NIHL focus on relatively distal aspects of eventual cell death, such as apoptosis; however, these biological processes may be too late to be effectively controlled. In contrast, the early stages of the UPR, including regulation of $\mathrm{ER} \mathrm{Ca}^{2+}$, may be a more viable site for intervention; we demonstrate in this study that upregulation of UPR genes occurs within 2 hours of noise exposure. TMTC4, as a key player in the early stages of cochlear trauma, may be a promising and specific target for drug development for hearing loss.

\section{Methods}

Study design. There were 4 primary goals in this study. First, to evaluate the phenotype of hearing loss in Tmtc4-KO mice and to demonstrate that $\mathrm{KO}$ of Tmtc4 in 2 independent genetic backgrounds still yielded the same hearing deficit. Second, to assess what impact absence of Tmtc4 has on ER $\mathrm{Ca}^{2+}$ signaling and downstream activation of the UPR. Third, to test whether Tmtc4 interacts with ER membrane proteins to further strengthen this hypothesis. Fourth, to assess whether modifying the UPR signaling pathway can diminish the observed hearing loss in either a genetic or environmentally acquired hearing-loss model (noise exposure). In all experiments, animals were randomly 
A

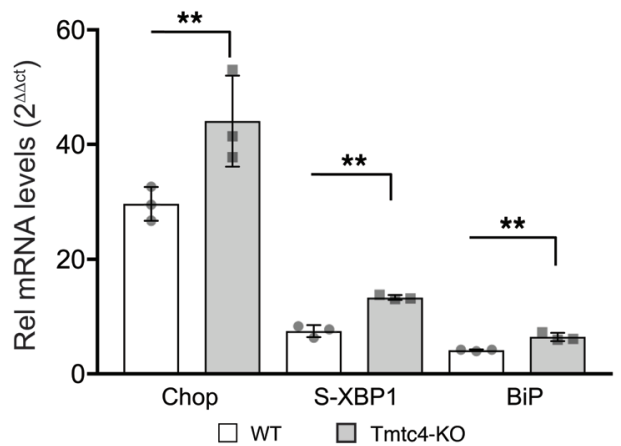

B
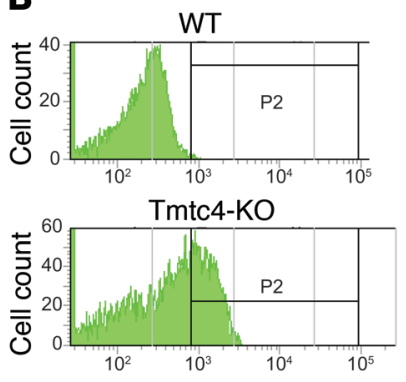

C

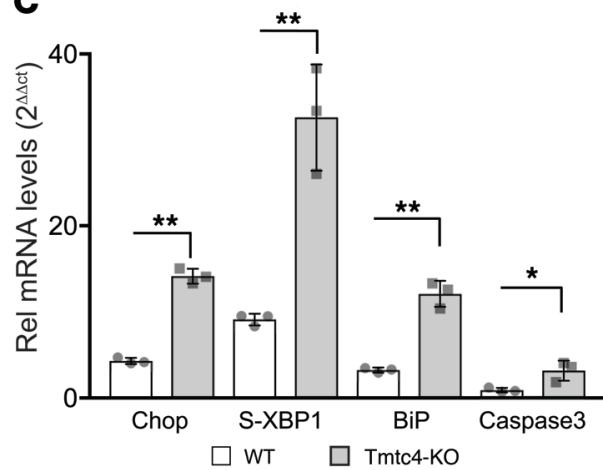

Figure 7. Upregulation of the UPR in Tmtc4-KO cells and cochleae. (A) mRNA levels of 3 genes (Chop, S-XBP1, and BiP) that represent the 3 principal arms of the UPR were measured in fibroblasts treated with the SERCA2B inhibitor thapsigargin (TG). Treatment of fibroblasts from WT and Tmtc4-KO mice with TG elicited an exaggerated upregulation of all 3 UPR effectors in KO compared with WT cells, as measured relative to expression of GAPDH and to unexposed $\mathrm{KO}$ and WT fibroblasts, respectively, by the $2^{\Delta \Delta c t}$ method. (B) FACS sorting of KO and WT cells positive for staining with antibody against Chop shows a greater percentage of KO cells (29.9\% vs $0.5 \%$ of WT cells) positive for Chop expression. P2: gating set at 3 SD from the mean fluorescence for WT cells. (C) mRNA levels of Chop, S-XBP1, and BiP were also upregulated to a greater extent in Tmtc4-KO cochlear explant cultures treated with TC compared with WT control cultures. TG treatment also induced apoptosis in KO cochlear cultures, as measured by the expression of caspase 3 . Data represent the mean and SD of 3 independent experiments; culture dishes of confluent fibroblasts (A) or single cochlear cultures (C). ${ }^{*} P<0.01 ;{ }^{*} P<0.001$ by 1 -way ANOVA followed by Tukey's multiple comparisons test.

assigned to experimental or treatment groups and matched for age, sex, and littermate controls. All measurements (hearing thresholds, $\mathrm{Ca}^{2+}$ signaling, biochemistry, and histological analyses) were carried out blind to genotype and treatment groups.

Mouse genetic models. ES cells were obtained from the KO mouse project repository (www.komp.org) that contained a nonconditional genomic disruption of Tmtc4 via homologous recombination (15) beginning at the $5^{\prime}$ end of exon 1 through the $3^{\prime}$ end of exon 3 , replaced with a cassette containing $\beta$-gal and neomycin (Supplemental Figure 1A). We confirmed that the ES cells carried this deletion of Tmtc4 by PCR; these cells were then injected into the blastocysts of C57BL/6J mice. Chimeric pups were tested via coat color and testing of tail DNA. Vertical transmission was confirmed in the subsequent generation through testing of offspring. KO and Het offspring were viable and KO/Het/WT mice were born with the predicted Mendelian frequencies. Tmtc4-KO mice were then crossed onto an FVB/NJ (FVB) background to avoid the age-related hearing loss (Ahl) phenotype associated with the C57BL/6J (B6) line. Chop-KO mice were obtained from the Jackson Laboratory (Ddit $3^{\text {tm2.1Dron}} ;$ strain 005530).

Tmtc4-KO fibroblasts and transgenic cell lines. For in vitro evaluation of the pathophysiologic effects of Tmtc4 deficiency, we generated stable fibroblast cell lines from P3 WT and Tmtc4-KO mice. Briefly, a skin sample from the axillary region is collected and cut into 1-mm pieces. Samples are then digested in 0.14 Wunsch units/ml Liberase Blendzyme 3 (Roche) in $37^{\circ} \mathrm{C}$ for 30 minutes. After washing in complete media, the sample pellets were resuspended in DMEM/F12 media with $10 \% \mathrm{FBS}$, transferred to a $10-\mathrm{cm}$ tissue-culture dish and placed in a tissue-culture incubator at $37^{\circ} \mathrm{C} / 5 \% \mathrm{CO}_{2}(49)$.

In order to perform further biochemical investigation of TMTC4 expression and localization, we performed transfection of HEK 293/ T17 cells (CRL-11268, ATCC). HEK cells were transfected with pIRESEGFP-puro (45567, Addgene), into which the human cDNA for a cMyctagged TMTC4 (TMTC4-Myc) was subcloned. For stable transfection, HEK single-cell clones were picked and expanded after puromycin selection. We also generated a TMTC4-KO HEK cell line to use as a negative control for these cell-line based experiments (Supplemental Figure 1, B and C). The targeted DNA probe on TMTC4 exon1 was selected by the CRISPR Design Tool (http://tools.genome-engineering. org) and appended onto the U6 primer. HEK cells were cotransfected with the Cas9 expression plasmid (pSpCas9) and a PCR-amplified U6-driven sgRNA expression cassette. The transfected cells were clonally isolated and expanded for genotyping analysis of deletion events. Loss of the TMTC4 protein in ER-enriched fractions was assessed by Western blotting (Supplemental Figure 1C).

Organotypic cochlear cultures. Organotypic cultures from P3-P5 mice of indicated genotypes were established as described (22). Briefly, P3 mice were euthanized and decapitated. Dissection was performed in dissecting solution (DS; $145 \mathrm{mM} \mathrm{NaCl}, 3 \mathrm{mM} \mathrm{KCl}, 0.25 \mathrm{mM}$ $\mathrm{CaCl}_{2}, 0.25 \mathrm{mM} \mathrm{MgCl}_{2}, 2 \mathrm{mM}$ Na-pyruvate, $5 \mathrm{mM}$ D-glucose, $10 \mathrm{mM}$ $\mathrm{Na}_{2} \mathrm{HPO}_{4}, \mathrm{pH}$ 7.35). The cochlear duct was isolated, opened, and plated on glass coverslips with Cell-Tak (Corning) with the apical surface of the epithelium facing up. Cultures were incubated overnight at $37^{\circ} \mathrm{C}$ and $5 \% \mathrm{CO}_{2}$ in DMEM-F12 plus $10 \% \mathrm{FBS}$ and $50 \mathrm{mg} / \mathrm{ml}$ ampicillin, and used for experiments after 24 hours in culture.

Analysis of TMTC4 localization. For immunofluorescent evaluation of TMTC4 localization, HEK cells were grown in DMEM supplemented with $10 \%$ fetal calf serum, $100 \mathrm{U} / \mathrm{ml}$ penicillin, and $100 \mathrm{mg} / \mathrm{ml}$ streptomycin, and incubated at $37^{\circ} \mathrm{C}$ in $5 \% \mathrm{CO}_{2}$. Cells were transfected with the aforementioned TMTC4-Myc plasmid and selected with puromycin. For staining, cells were dissociated into single cell culture and incubated on glass coverslips. After 48 hours, the cells were fixed with $4 \%$ paraformaldehyde in PBS for 10 minutes at RT followed by permeabilization with methanol for 10 minutes at $20^{\circ} \mathrm{C}$. Slides were stained with the indicated primary antibody followed by staining with the appropriate Alexa Fluor 488 or 594 secondary antibodies (A11029 and A11012, Thermo Fisher Scientific). Slides were rinsed and mounted with coverslips using VectaShield (Vector Laboratories). Images were obtained with a Carl Zeiss Axio Observer inverted microscope. The primary antibodies used were against human 
A
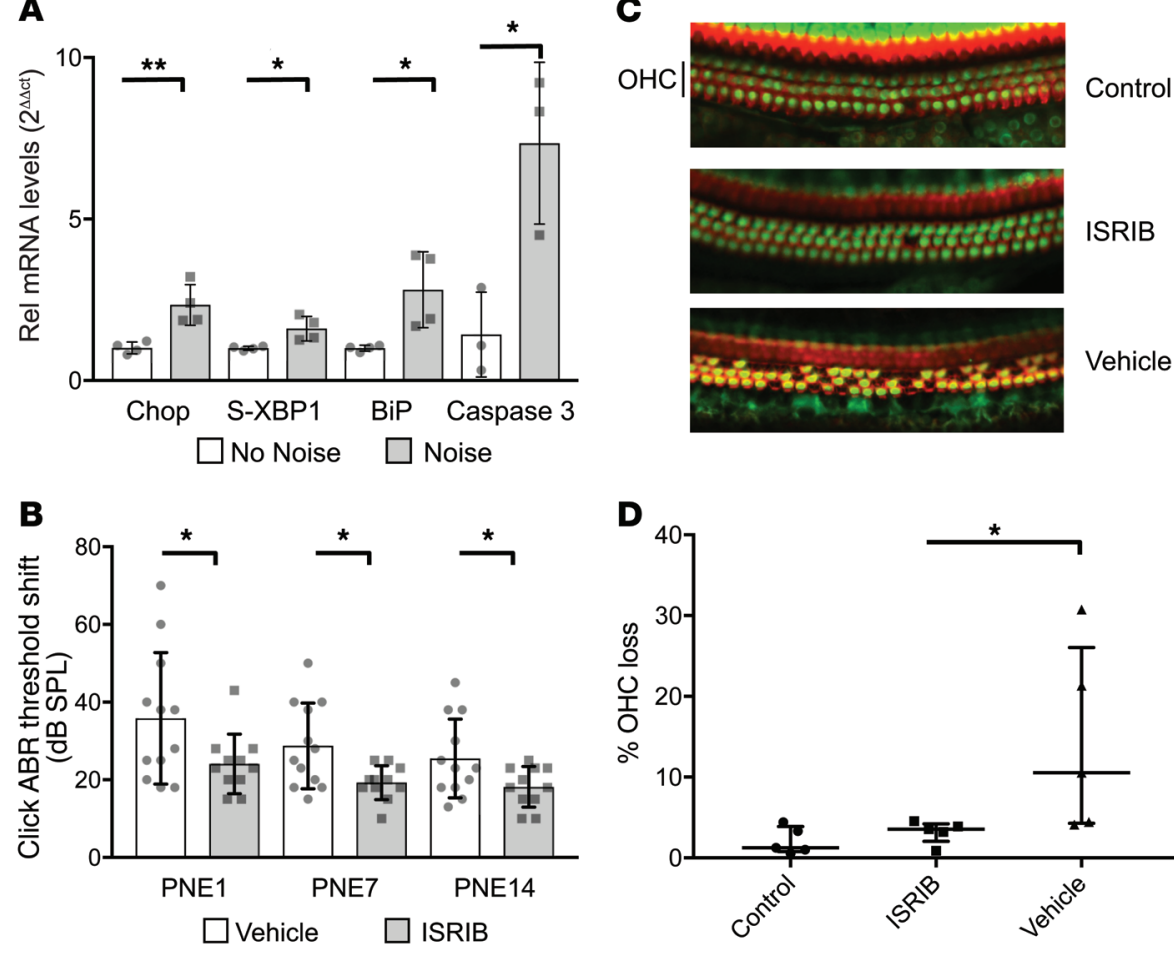

Figure 8. UPR in noise-exposed WT mice. (A) Eight-week-old WT FVB mice were exposed to 8-16 $\mathrm{kHz}$ octave-band white noise at $106 \mathrm{~dB}$ SPL for 120 minutes. Cochleae were removed 2 hours after exposure (Noise) or unexposed (No Noise). mRNA expression of Chop, S-XBP1, BiP, and caspase 3 is presented relative to expression of GAPDH and the average expression levels of control, unexposed cochleae by the $2^{\Delta \Delta c t}$ method. Data represent the mean and SD of 4 (Chop, S-XBP1, and BiP) or 3 (caspase) cochleae in each condition. ${ }^{*} P<0.05 ;{ }^{*} P<0.01$ by 1 -way ANOVA followed by Tukey's multiple comparisons test. (B) Eight-week-old WT FVB mice were exposed to noise, and ABR thresholds were measured at 1,7 , and 14 days post-noise exposure (PNE). Intraperitoneal injection of ISRIB ( $n=11) 2$ hours prior to noise exposure attenuated the hearing loss observed in vehicle-injected mice $(n=12)$. Data represent mean and SD. ${ }^{*} P<0.05$ by 1 -way ANOVA followed by Tukey's multiple comparisons test. (C) Whole-mount immunohistochemical staining for Myo7a (green, hair cell marker) and actin (red, phalloidin) demonstrates 3 intact rows of outer hair cells (OHC) in control, nonexposed mice (top); significant $\mathrm{OHC}$ loss in mice exposed to noise and treated with vehicle (bottom); and minimal OHC loss in ISRIB-treated mice exposed to noise (middle). Images representative of 5 cochleae for each condition. Original magnification $\times 60$. (D) $\mathrm{OHC}$ number was quantified in the mid-basal turn of the cochlea $(n=5$ cochleae for each condition, data presented as individual values with median and interquartile range), demonstrating significantly greater $\mathrm{OHC}$ loss in vehicle-treated animals exposed to noise (Vehicle), compared with those treated with ISRIB, or non-noise-exposed controls. ${ }^{*} P<0.05$ by Mann-Whitney $U$ rank-sum test.

TMTC4 (HPA016489, Sigma-Aldrich), SERCA2B (ab2861, Abcam), GRP94 (ab2791, Abcam), and GM130 (610822, BD Biosciences).

Subcellular localization and coimmunoprecipitation were performed in WT HEK cells, and in HEK cells stably transfected with TMTC4-Myc fusion plasmid, or with empty plasmid. Cells were suspended in hypotonic extraction buffer (10 mM HEPES, pH 7.8, $25 \mathrm{mM}$ $\mathrm{KCl}, 1 \mathrm{mM}$ EGTA, and protease inhibitor) for 30 minutes at $4^{\circ} \mathrm{C}$ and centrifuged at $600 \mathrm{~g}$ for 5 minutes. For nonnuclear whole-cell lysate, the cell pellet was suspended in isotonic buffer (ISO; $10 \mathrm{mM}$ HEPES, pH 7.8, $250 \mathrm{mM}$ sucrose, $25 \mathrm{mM} \mathrm{KCl}, 1 \mathrm{mM}$ EGTA, and protease inhibitor) and transferred to a 7-ml Dounce homogenizer. Cells were broken with 10 strokes of the Dounce homogenizer and the percentage of broken cells checked by light microscopy. The homogenate was centrifuged at $650 \mathrm{~g}$ for 10 minutes twice and the postnuclear supernatant (PNS) collected. For isolation of ER-enriched fractions, the cell pellet was homogenized in ice-cold buffer with 225 mM mannitol, $75 \mathrm{mM}$ sucrose, $0.1 \mathrm{mM}$ EGTA, and $30 \mathrm{mM}$ Tris- $\mathrm{HCl}, \mathrm{pH}$ 7.4. The homogenate was subjected to a series of centrifugation steps to achieve an ER-enriched cellular subfraction as previously described (50).

For coimmunoprecipitation experiments, $500 \mu \mathrm{g}$ of the aforementioned PNS homogenate was incubated with agarose beads coupled with antibodies against TMTC4 (HPA016489, MilliporeSigma), or with beads alone as a negative control, and placed in spin columns (Pierce Biotechnology). Proteins that bound to this linked antibody (the eluate) were detected by immunoblotting with antibodies against c-Myc, SERCA2B, and calnexin. The Odyssey infrared system (Li-Cor Biosciences) was used for qualitative and quantitative Western blot image analysis. Western blotting was performed using an Odyssey infrared imaging system (LiCor Biosciences). Briefly, total protein was isolated from fibroblast cells, separated by electrophoresis on $4 \%-20 \%$ gradient tri-glycine gels (Bio-Rad), and then transferred to PVDF (MiliporeSigma). Antibodies used for detection were as follows: primary antibodies mouse anti-Chop (Ma1-250, Thermo Fisher Scientific), rabbit anti-DR5 (ab8416, Abcam), rabbit anti-caspase 8 (9429, Cell Signaling Technology), and mouse anti-beta actin (3700, Cell Signaling Technology); and infrared-labeled secondary antibodies goat anti-mouse IRDye 800CW (926-32210, Li-Cor Biosciences) or goat anti-rabbit IRDye 680RD (926-68071, Li-Cor Biosciences). The bound complex was detected using the Odyssey infrared imaging system, and images were analyzed using the Odyssey Application Software, version 1.2 (Li-Cor Biosciences).

The ER fraction and nonnuclear whole-cell lysate were also subjected to Western blot analysis as described above. The same approach of coimmunoprecipitation of TMTC4-associated proteins was used with human fetal brain tissue (approval for tissue donation was obtained from the UCSF Institutional Review Board, https://irb.ucsf.edu).

The glycosylation pattern of TMTC4 was assessed. HEK293T cells stably expressing TMTC4-Myc were lysed and homogenized as described above. The whole-cell nonnuclear lysate was isolated by centrifugation at $650 \mathrm{~g}$ for 10 minutes. Microsomes were pelleted after centrifugation at $100,000 \mathrm{~g}$ for 20 minutes. The pellets were suspended in isotonic buffer (ISO). An additional $1 \%$ of NP- 40 was added to the suspension containing $1 \mathrm{mg}$ of protein lysate to a final volume to $400 \mu \mathrm{l}$. The lysate was precleared with empty agarose beads. To extract TMTC4 and bound proteins, this cleared lysate was incubated overnight with antibody against c-Myc coupled to agarose beads. The next day these beads were washed 3 times with ISO buffer and glycosylation assays were performed using endoglycosidase $\mathrm{H}$ (Endo $\mathrm{H}$ ) 
A

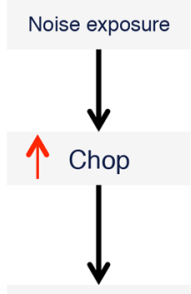

Hearing loss

B

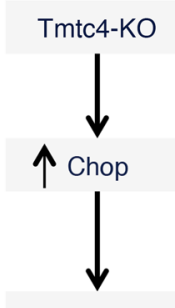

Hearing loss

c

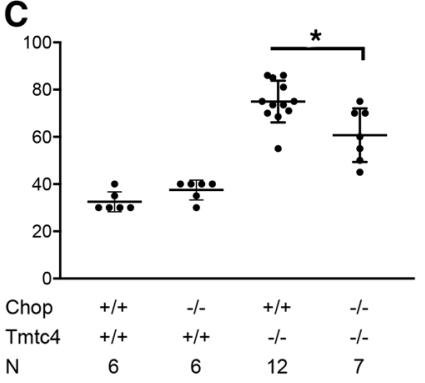

Figure 9. Inverse complementation assay. (A) As demonstrated in Figure 8, noise exposure induces expression of the proapoptotic UPR modulator Chop, and pharmacologic inhibition of Chop expression with ISRIB via ATF4 attenuates NIHL. (B) In the genetic model of Tmtc4 deficiency, Tmtc4-KO cochlea demonstrate upregulation of Chop. In an inverse complementation model, we generated double $\mathrm{KO}$ mice with both Tmtc4 and Chop deficiency and hypothesized that prevention of Chop expression would attenuate progressive postnatal hearing loss. (C) ABR thresholds were measured for mice of the indicated genotypes for Tmtc4 and Chop. Tmtc4 $4^{-/-}$mice were all tested at P19; Tmtc4 ${ }^{+/+}$mice were all tested at P39 to ensure no longterm hearing loss associated with Chop deficiency alone. Tmtc $4^{-/-}$mice showed elevated ABR thresholds compared with all Tmtc $4^{+/+}$mice; among the $\mathrm{Tmtc4}^{-/-}$mice, Chop -/- $^{-}$genotype was associated with improved hearing compared with Chop ${ }^{+/+}$mice. ${ }^{*} P<0.01$ by unpaired 2 -tailed $t$ test. Data represent mean and SD.

or peptide $\mathrm{N}$-glycosidase F (PNGase F) according to the New England Biolabs protocol. Finally, reducing sample buffer was added to all samples, and analyzed by Western blot with mouse antibody against c-Myc (3700, Cell Signaling Technology).

UPR gene expression analysis. Expression of mRNA for ER stress markers (CHOP, S-XBP1, and BiP) was quantified in cultured fibroblasts, cell lines, organotypic cochlear cultures, and adult mouse cochleae. Tmtc4-KO and WT cultured fibroblasts or organotypic cochlear cultures were treated with DMEM-F12 or DS, respectively, or with media containing $0.5 \mu \mathrm{M}$ thapsigargin, for 2 hours and then harvested onto dry ice. Eight-week-old WT FVB mice (001800, Jackson laboratories) were exposed to $106 \mathrm{~dB}$ octave-band noise for 2 hours.
Two hours after completion of noise exposure, animals were euthanized and cochleae harvested onto dry ice.

Total RNA was isolated using the Purolink RNA mini kit (Life Technologies), and $1 \mu \mathrm{g}$ total RNA was used for first-strand cDNA synthesis using the SuperScript IV VILO master mix (Invitrogen). Realtime PCR was performed by 7900 HT FAST real-time PCR system (Applied Biosystems) with a SYBR green I mix. The primers for mRNA to ER stress markers are as follows: Chop, CCACCACACCTGAAAGCAGAA and AGGTGAAAGGCAGGGACTCA; $S$-XBP1, CTGAGTCCGAATCAGGTGCAG and GTCCATGGGAAGATGTTCTGG; and BiP, TTCAGCCAATTATCAGCAAACTCT and TTTTCTGATGTATCCTCTTCACCAGT. Additional quantification was performed for caspase 3 (GGAGAACAACAAAACCTCAGTGGATTC and CATTCCAGTGCTCTTATGGAAGTTCTTATTATTA). Relative quantification by the $2^{-\triangle \Delta C T}$ method was used for analysis of real-time PCR data as previously described (51). Briefly, this method directly uses the $C T$ information generated from a qPCR system to calculate relative gene expression in target and reference samples, using an internal control or housekeeping gene (GAPDH) as the normalizer. The first $\triangle C T$ is the difference in threshold cycle between the target and internal control genes; the $\triangle \triangle C T$ is the difference in $\triangle C T$ as described in the above formula between the target and reference samples. For this study, the reference sample consisted of unexposed cells, cochlear cultures, or animals.

For flow cytometric analysis of Chop expression, WT or Tmtc4-KO fibroblast cells were collected by trypsin digestion to obtain a single cell suspension, quenched by washing with complete media and centrifuged. The pellet was fixed in $4 \%$ formaldehyde in PBS for 10 minutes at $37^{\circ} \mathrm{C}$ followed by permeabilization in ice-cold $100 \%$ methanol for 30 minutes. Cells were washed in PBS with 0.5\% BSA and labeled with mouse anti-Chop antibody (MA1-250, Thermo Fisher Scientific) or with mouse isotype control (ab37355, Abcam) for 1 hour at RT. The cells were then labeled with goat anti-mouse IgG2b secondary antibody and Alexa Fluor 594 (A21145, Thermo Fisher Scientific) for 30 minutes. Labeled cells were resuspended in $0.5 \mathrm{ml}$ PBS and run on a BD FACSAria II flow cytometer using FACSDiva software (BD Biosciences). For quantitative comparison of Chop expression between WT and Tmtc4-KO cells, the gating threshold was set at a level 3 standard deviations above the mean fluorescence of WT stained cells.

Auditory testing and noise exposure. Hearing was tested in mice, including B6 and FVB Tmtc4-KO/het, B6 Chop/Tmtc4-DKO, and FVB WT mice in vivo by measuring auditory brainstem response (ABR) thresholdsin response to broadband clicks and tone pips, and distortionproduct otoacoustic emissions (DPOAEs) in the sound field using a standard commercial system (RZ6, Tucker-Davis Technologies) in a soundproof chamber $(13,22)$. We examined the effect of acoustic overstimulation in 8-week-old WT FVB mice of both sexes in vivo. Hearing was tested at baseline and 1, 7, and 14 days after noise exposure. Animals were exposed to $106 \mathrm{~dB}$ octave-band $(8-16 \mathrm{kHz})$ white noise for 120 minutes in a custom-built, calibrated, reverberant sound chamber (22). Animals were not anesthetized, in order to avoid the effects of general anesthesia on sound-induced threshold shifts (52). To assess the effect of UPR modulation on NIHL, we pretreated animals with ISRIB prior to noise exposure. Mice were injected intraperitoneally 3 hours prior to noise exposure with a single dose of $2.5 \mathrm{mg} /$ kg ISRIB (MilliporeSigma) or vehicle (50\% DMSO, 50\% PEG-400). Noise exposure and ABR testing proceeded as described above. All 
ABR measurements were performed by an investigator blinded to the treatment (ISRIB or vehicle).

$\mathrm{Ca}^{2+}$ imaging. To evaluate intracellular $\mathrm{Ca}^{2+}$ concentration $\left[\mathrm{Ca}^{2+}\right]_{\mathrm{i}}$, ratiometric imaging was performed using FURA-2-AM (Thermo Fisher Scientific), a cell-permeant dye, in Tmtc4-KO and WT neonatal organotypic cultures (22). For FURA-2, the ratio of emission stimulated upon 340-nm and 387-nm excitation is proportional to $\left[\mathrm{Ca}^{2+}\right]_{\mathrm{i}}$, owing to different $\mathrm{Ca}^{2+}$-dependence of its excitation spectra in response to 340-nm and 387-nm light, the ratio of emission stimulated upon 340$\mathrm{nm}$ and $387-\mathrm{nm}$ excitation is proportional to $\left[\mathrm{Ca}^{2+}\right]$ and independent of dye concentration, facilitating stable imaging of intracellular $\left[\mathrm{Ca}^{2+}\right]$. Cochleae were loaded by incubation with $16 \mu \mathrm{M}$ FURA-2-AM in DS plus $0.01 \mathrm{wt} / \mathrm{vol}$ Pluronic F-127 and $250 \mu \mathrm{M}$ sulfinpyrazone for 15 minutes at RT, followed by a 10 -minute wash in DS at $37^{\circ} \mathrm{C}$ for deesterification. Alternating excitation at $340 \mathrm{~nm}$ and $387 \mathrm{~nm}$ was performed using a high-speed fluorescence light source, filter wheel, and shutter (Lambda XL, Sutter Instruments), and emission at $510 \mathrm{~nm}$ was recorded using a high-speed CMOS camera (ORCA-Flash4.0, Hamamatsu). Imaging was performed with a $\times 60,0.80 \mathrm{NA}$, waterimmersion objective on an upright microscope (BX-51, Olympus). A 10-ms exposure time at 3-second intervals was used to avoid photobleaching. The ratio of emission stimulated upon 340- and 387-nm excitation (proportional to $\left[\mathrm{Ca}^{2+}\right]_{\mathrm{i}}$ ), and was measured in regions of interest (ROI) using MetaFluor (Molecular Devices). A total of 225 ROIs were defined on a $100 \mu \mathrm{m}^{2}$ grid encompassing the entire cochlear epithelium, such that each ROI encompassed a $7 \mathrm{x} 7 \mu \mathrm{m}^{2}$ area slightly larger than a single cell. Peaks in cytosolic $\mathrm{Ca}^{2+}$ levels were detected using a custom peak detector (MatLab), which permitted the extraction of individual peak height (increase in fluorescence ratio from baseline to peak maximum), individual peak decay time (time from peak maximum to half-maximal value), and aggregate peak frequency (number of peaks per minute). Cultures were imaged and recorded for a total of 30 minutes. Thapsigargin (MilliporeSigma) and CDN1163 (Tocris Bioscience), respectively an inhibitor and activator of SERCA2b, were used to modulate $\mathrm{Ca}^{2+}$ dynamics. For drug application, baseline recording was obtained for 5 minutes in DS, followed by bath application of DS plus drug(s) as indicated and further imaging for 30 minutes. All parameters measured in the presence of drug were normalized to the parameters measured during the baseline acquisition period.

Tmtc4 expression and deficiency in the cochlea. Morphological differences attributable to Tmtc4 deficiency were examined by light microscopy of stained thin sections. Tmtc4-KO mice and WT littermates at indicated ages were anesthetized, and their cochleae were isolated, dissected, perfused through the round and oval windows with a solution of $2.5 \%$ paraformaldehyde and $1.5 \%$ glutaraldehyde in $0.1 \mathrm{M}$ phosphate buffer $(\mathrm{PB})$ at $\mathrm{pH} 7.4$, and finally incubated in the same fixative overnight at $4^{\circ} \mathrm{C}$. The cochleae were rinsed with $0.1 \mathrm{M} \mathrm{PB}$ and postfixed in $1 \%$ osmium tetroxide for 2 hours. The cochleae were subsequently immersed in 5\% EDTA for decalcification. The decalcified cochleae were dehydrated with ethanol and propylene oxide and then embedded in Araldite 502 resin (Electron Microscopy Sciences). The cochleae were then sectioned at $5-\mu \mathrm{m}$ thickness parallel to the cochlea modiolus. The sections were stained with toluidine blue and mounted with Permount (Thermo Fisher Scientific) on microscope slides and visualized with a Leica microscope.

Cell-specific expression of Tmtc4 was evaluated with $\beta$-gal protein staining. Tmtc4-Het mouse littermates at P21 were anesthetized, and their cochleae were visualized, dissected, and then perfused through the oval and round windows by $4 \%$ paraformaldehyde in 0.1M PBS, $\mathrm{pH}$ 7.4. The isolated cochleae were then incubated in the same fixative for 2 hours. After fixation, cochleae were rinsed with PBS and immersed in 5\% EDTA in 0.1 M PBS for decalcification. Decalcified cochleae (24-36 hours) were embedded in paraffin and 5- $\mu$ m-thick slices cut in a microtome for immunohistochemistry. These sections were incubated in $\beta$-gal antibody (A11132, Thermo Fisher Scientific) overnight, rinsed with PBS, and incubated with biotinylated secondary antibody (BA-110, Vector Laboratories) for 1 hour. For peroxidase detection, VECTASTAIN Elite ABC kit (PK-6100, Vector Laboratories) and ImmPact DAB kit (SK-4105, Vector Laboratories) were used according to the manufacturer's instructions.

Evaluation of hair cell loss was performed with whole-mount cochlear immunohistochemistry. Cochleae from Tmtc4-KO mice and WT littermates at varying time points were isolated, fixed, and decalcified as described above. Following decalcification, the otic capsule was then removed, followed by removal of the lateral wall, Reissner's membrane, and tectorial membrane. The remaining organ of Corti was incubated with anti-myosin VIIa antibody (a hair cell-specific marker; 1:50 dilution in PBS; 25-6790, Proteus Biosciences) and incubated overnight at $4^{\circ} \mathrm{C}$. Whole-mount cochleae were rinsed twice for 10 minutes with PBS and then incubated for 2 hours with a goat anti-rabbit IgG antibody conjugated to Cy2 (1:2,000 dilution in PBS; 111-165-003, Jackson ImmunoResearch). Whole mounts were rinsed in PBS twice for 15 minutes and incubated with rhodamine-phalloidin (stock solution of $200 \mathrm{U} / \mathrm{ml}$ methanol, diluted 1:100 in PBS for working solution) for 1 hour. Whole mounts were then rinsed with PBS, further microdissected into individual turns for surface preparation, and then exposed for 15 minutes at RT to fluorescent DAPI to mark nuclei. Cochlear whole mounts were rinsed in PBS and mounted on glass slides in antifade FluorSave reagent (34589, Calbiochem). Hair cells in the organ of Corti were visualized by confocal microscopy.

Expression levels of Tmtc isoforms 1-4 were compared in WT and Tmtc4-KO mouse cochleae at P21 using quantitative PCR. Preparation of RNA from mouse cochlea and qPCR were as described above. Primers used for respective genes were as follows: Tmtc1, ATGCTGGTGACCCGCGGGGA and CCTTGCCCCAGAAGTCGTTAG; Tmtc2, AGAGAGTGCAGAGTGGCCGGTAG and ATGATTGCAGAGTTGGTGAGCAGCG; Tmtc3, ATGCTTGAAGGGAAGATGGCTGATATTAACTT and GTGGCTTCTCTCCTCAGACATAGG; and Tmtc4, ATGGTTGAGCTGGATGCTGACTTGGA and GAAGTCATGGTGCCACAGGTCCC.

Study approval. The UCSF Institutional Animal Care and Use Committee (http://iacuc.ucsf.edu) approved the animal studies in the laboratories of DKC and EHS. No human studies were performed for this manuscript.

Statistics. For comparison between treatment groups for UPR gene expression, we used 1-way ANOVA followed by Tukey's multiple comparison tests. For pairwise comparison of ABR thresholds and DPOAE levels between groups of mice, and for comparisons of fluorescence levels and $\mathrm{Ca}^{2+}$ peak parameters between $\mathrm{Ca}^{2+}$ imaging experiments, we used unpaired 2-tailed Student's $t$ test. Unless otherwise mentioned, results are mean \pm SD with sample sizes and $P$ values between designated comparison groups as indicated in the figure legends, with a $P$ value less than 0.05 as significant, and lower $P$ values indicated for 
specific comparisons. Statistical analyses were performed with GraphPad and Matlab.

\section{Author contributions}

EHS, JL, LRL, OA, and DKC created the initial design. EHS and DKC oversaw experiments and ethics. JL, OA, SLR, CWM, IRM, and DKC contributed to the experiments. JL, OA, DKC, and EHS provided data and statistical analysis. EHS, JL, OA, LRL, SLR, and DKC drafted the manuscript.

\section{Acknowledgments}

We would like to thank Peter Walter and Robert Edwards for a careful reading of the manuscript. This work was supported in part by a grant from the National Institute of Neurological Disorders and Stroke (NINDS) to EHS (2R01NS058721), from the National Institute on Deafness and Other Communication Disorders (NIDCD) to DKC (R03DC015082), and from the NIH/National Center for Advancing Translational Sciences to EHS and DKC (UL1TR001872).

Address correspondence to: Elliott H. Sherr, Department of Neurology, UCSF, 675 Nelson Rising Lane, Room 214B, San Francisco, California 94158, USA. Phone: 415.514.9306; Email: elliott. sherr@ucsf.edu. Or to: Dylan K. Chan, Department of Otolaryngology - Head and Neck Surgery, UCSF, 513 Parnassus Avenue, Room 719, Box 3213, San Francisco, California 94143, USA. Phone: 415. 353.2757; Email: dylan.chan@ucsf.edu.
1. Kujawa SG, Liberman MC. Adding insult to injury: cochlear nerve degeneration after "temporary" noise-induced hearing loss. J Neurosci. 2009;29(45):14077-14085.

2. Spoendlin H. Primary structural changes in the organ of Corti after acoustic overstimulation. Acta Otolaryngol.1971;71(2):166-176.

3. Sha SH, Schacht J. Emerging therapeutic interventions against noise-induced hearing loss. Expert Opin Investig Drugs. 2017;26(1):85-96.

4. Fridberger A, Flock A, Ulfendahl M, Flock B. Acoustic overstimulation increases outer hair cell $\mathrm{Ca}^{2+}$ concentrations and causes dynamic contractions of the hearing organ. Proc Natl Acad Sci U S A . 1998;95(12):7127-7132.

5. Böttger EC, Schacht J. The mitochondrion: a perpetrator of acquired hearing loss. Hear Res. 2013;303:12-19.

6. Bielefeld EC, Kopke RD, Jackson RL, Coleman JK, Liu J, Henderson D. Noise protection with $\mathrm{N}$-acetyl-l-cysteine (NAC) using a variety of noise exposures, NAC doses, and routes of administration. Acta Otolaryngol. 2007;127(9):914-919.

7. Pourbakht A, Yamasoba T. Ebselen attenuates cochlear damage caused by acoustic trauma. Hear Res. 2003;181(1-2):100-108.

8. Wang J, Van De Water TR, Bonny C, de Ribaupierre F, Puel JL, Zine A. A peptide inhibitor of c-Jun $\mathrm{N}$-terminal kinase protects against both aminoglycoside and acoustic trauma-induced auditory hair cell death and hearing loss. J Neurosci. 2003;23(24):8596-8607.

9. Uemaetomari I, Tabuchi K, Nakamagoe M, Tanaka S, Murashita H, Hara A. L-type voltagegated calcium channel is involved in the pathogenesis of acoustic injury in the cochlea. Tohoku JExp Med. 2009;218(1):41-47.

10. Shen H, et al. Prophylactic and therapeutic functions of T-type calcium blockers against noise-induced hearing loss. Hear Res. 2007;226(1-2):52-60.

11. Kopke R, et al. Efficacy and safety of Nacetylcysteine in prevention of noise induced hearing loss: a randomized clinical trial. Hear Res. 2015;323:40-50.

12. Suckfuell M, Canis M, Strieth S, Scherer H, Haisch A. Intratympanic treatment of acute acoustic trauma with a cell-permeable JNK ligand: a prospective randomized phase I/II study. Acta Otolaryngol. 2007;127(9):938-942.
13. Akil O, et al. Restoration of hearing in the VGLUT3 knockout mouse using virally mediated gene therapy. Neuron. 2012;75(2):283-293

14. Delmaghani S, et al. Hypervulnerability to sound exposure through impaired adaptive proliferation of peroxisomes. Cell. 2015;163(4):894-906.

15. Valenzuela DM, et al. High-throughput engineering of the mouse genome coupled with high-resolution expression analysis. Nat Biotechnol. 2003;21(6):652-659.

16. Keithley EM, Canto C, Zheng QY, FischelGhodsian N, Johnson KR. Age-related hearing loss and the ahl locus in mice. Hear Res. 2004;188(1-2):21-28.

17. Hickox AE, et al. Global analysis of protein expression of inner ear hair cells. JNeurosci. 2017;37(5):1320-1339.

18. Scheffer DI, Shen J, Corey DP, Chen ZY. Gene expression by mouse inner ear hair cells during development. J Neurosci. 2015;35(16):6366-6380.

19. Runge CL, et al. Association of TMTC2 with human nonsyndromic sensorineural hearing loss. JAMA Otolaryngol Head Neck Surg. 2016;142(9):866-872.

20. Blom N, Sicheritz-Pontén T, Gupta R, Gammeltoft S, Brunak S. Prediction of post-translational glycosylation and phosphorylation of proteins from the amino acid sequence. Proteomics. 2004;4(6):1633-1649.

21. Henderson MJ, et al. A low affinity GCaMP3 variant (GCaMPer) for imaging the endoplasmic reticulum calcium store. PLoS One. 2015;10(10):e0139273.

22. Chan DK, Rouse SL. Sound-induced intracellular $\mathrm{Ca}^{2+}$ dynamics in the adult hearing cochlea. PLoS One. 2016;11(12):e0167850.

23. Mammano F. ATP-dependent intercellular $\mathrm{Ca}^{2+}$ signaling in the developing cochlea: facts, fantasies and perspectives. Semin Cell Dev Biol. 2013;24(1):31-39.

24. Gale JE, Piazza V, Ciubotaru CD, Mammano F. A mechanism for sensing noise damage in the inner ear. Curr Biol. 2004;14(6):526-529.

25. Lytton J, Westlin M, Hanley MR. Thapsigargin inhibits the sarcoplasmic or endoplasmic reticulum Ca-ATPase family of calcium pumps. J Biol Chem. 1991;266(26):17067-17071.

26. Kang S, et al. Small molecular allosteric activator of the Sarco/Endoplasmic reticulum $\mathrm{Ca}^{2+}$-ATPase (SERCA) attenuates diabetes and metabolic dis- orders. J Biol Chem. 2016;291(10):5185-5198.

27. Walter $\mathrm{P}$, Ron $\mathrm{D}$. The unfolded protein response: from stress pathway to homeostatic regulation. Science. 2011;334(6059):1081-1086.

28. $\mathrm{Lu} \mathrm{M}$, et al. Opposing unfolded-protein-response signals converge on death receptor 5 to control apoptosis. Science. 2014;345(6192):98-101.

29. Sidrauski C, et al. Pharmacological dimerization and activation of the exchange factor eIF2B antagonizes the integrated stress response. Elife. 2015;4:e07314

30. Sidrauski C, et al. Pharmacological brake-release of mRNA translation enhances cognitive memory. Elife. 2013;2:e00498.

31. Liu SH, et al. C/EBP homologous protein (CHOP) deficiency ameliorates renal fibrosis in unilateral ureteral obstructive kidney disease. Oncotarget. 2016;7(16):21900-21912.

32. Noh MR, Kim JI, Han SJ, Lee TJ, Park KM. C/EBP homologous protein (CHOP) gene deficiency attenuates renal ischemia/reperfusion injury in mice. Biochim Biophys Acta. 2015;1852(9):1895-1901.

33. Nam DH, et al. CHOP deficiency prevents methylglyoxal-induced myocyte apoptosis and cardiac dysfunction. JMol Cell Cardiol. 2015;85:168-177.

34. Cai T, Jen HI, Kang H, Klisch TJ, Zoghbi HY, Groves AK. Characterization of the transcriptome of nascent hair cells and identification of direct targets of the Atoh1 transcription factor. J Neurosci. 2015;35(14):5870-5883.

35. Guillen-Ahlers H, et al. TMTC2 variant associated with sensorineural hearing loss auditory neuropathy spectrum disorder in a family dyad [published online ahead of print April 19, 2018]. Mol Genet Genomic Med. https://doi.org/10.1002/mgg3.397.

36. Bowl MR, et al. A large scale hearing loss screen reveals an extensive unexplored genetic landscape for auditory dysfunction. Nat Commun. 2017;8(1):886.

37. Tao J, Petrova K, Ron D, Sha B. Crystal structure of P58(IPK) TPR fragment reveals the mechanism for its molecular chaperone activity in UPR. JMol Biol. 2010;397(5):1307-1315

38. Sunryd JC, Cheon B, Graham JB, Giorda KM, Fissore RA, Hebert DN. TMTC1 and TMTC2 are novel endoplasmic reticulum tetratricopeptide repeat-containing adapter proteins involved in calcium homeostasis. J Biol Chem. 2014;289(23):16085-16099. 
39. Lau W, Andrew T, Maniatis N. High-resolution genetic maps identify multiple type 2 diabetes loci at regulatory hotspots in African Americans and Europeans. Am J Hum Genet. 2017;100(5):803-816.

40. Li L, et al. Identification of type 2 diabetes subgroups through topological analysis of patient similarity. Sci Transl Med. 2015;7(311):311ra174.

41. Larsen ISB, et al. Discovery of an O-mannosylation pathway selectively serving cadherins and protocadherins. Proc Natl Acad Sci U S A. 2017;114(42):11163-11168.

42. Blanco-Sánchez B, Clément A, Fierro J, Washbourne P, Westerfield M. Complexes of Usher proteins preassemble at the endoplasmic reticulum and are required for trafficking and ER homeostasis. Dis Model Mech. 2014;7(5):547-559.

43. Perera N, Miller JL, Zitzmann N. The role of the unfolded protein response in dengue virus patho- genesis. Cell Microbiol. 2017;19(5):e12734.

44. Yeganeh B, et al. Hepatitis B and C virusinduced hepatitis: Apoptosis, autophagy, and unfolded protein response. World J Gastroenterol. 2015;21(47):13225-13239.

45. Zatyka M, et al. Sarco(endo)plasmic reticulum ATPase is a molecular partner of Wolfram syndrome 1 protein, which negatively regulates its expression. Hum Mol Genet. 2015;24(3):814-827.

46. Celli A, et al. SERCA2-controlled $\mathrm{Ca}^{2}+$ dependent keratinocyte adhesion and differentiation is mediated via the sphingolipid pathway: a therapeutic target for Darier's disease. J Invest Dermatol. 2012;132(4):1188-1195.

47. Godic A, Korosec B, Miljković J, Kansky A, Glavac D. Four novel ATP2A2 mutations in Slovenian patients with Darier disease. JAm Acad Dermatol. 2010;62(5):819-823.

48. Wang $\mathrm{W}$, et al. Impaired unfolded protein response in the degeneration of cochlea cells in a mouse model of age-related hearing loss. Exp Gerontol. 2015;70:61-70.

49. Seluanov A, Vaidya A, Gorbunova V. Establishing primary adult fibroblast cultures from rodents. JVis Exp. 2010;(44):2033.

50. Wieckowski MR, Giorgi C, Lebiedzinska M, Duszynski J, Pinton P. Isolation of mitochondriaassociated membranes and mitochondria from animal tissues and cells. Nat Protoc. 2009;4(11):1582-1590.

51. Livak KJ, Schmittgen TD. Analysis of relative gene expression data using real-time quantitative PCR and the 2(-Delta Delta C(T)) Method. Methods. 2001;25(4):402-408.

52. Chung JW, et al. The effect of isoflurane, halothane and pentobarbital on noiseinduced hearing loss in mice. Anesth Analg. 2007;104(6):1404-1408, table of contents. 